\title{
Properties of spontaneous contractions and their modulation by transmural nerve stimulation in circular smooth muscle isolated from the pacemaker area in the flexure region of the guinea-pig colon
}

\author{
Hiroyuki FujImoto ${ }^{1}$, Yuhsuke SHIGEMASA ${ }^{1}$ and Hikaru SuZukI ${ }^{1}$ \\ ${ }^{1}$ Department of Cell Physiology, Nagoya City University Medical School, Japan
}

Received September 28, 2010; Accepted October 31, 2010

\begin{abstract}
The properties of spontaneous contractions and their modulation by transmural nerve stimulation (TNS) were investigated in circular smooth muscle preparations isolated from the "pacemaker area" in the flexure region of the guinea-pig colon. Four types of preparation were made; mucosal layer removed preparations, mucosal and submucosal layer removed preparations (submucosal layer removed preparations), longitudinal muscle removed preparations, and preparations with all layers attached (intact preparations). Intact and mucosal layer removed preparations periodically generated two types of phasic contractions; large contractions at a low frequency (about 2 times per min) and small contractions at a higher frequency (about 13 times per min). Submucosal layer removed preparations only produced large contractions, while longitudinal muscle removed preparations only produced small contractions. Both the amplitude and frequency of the large contractions were significantly inhibited by atropine, but were not markedly modulated by $\mathrm{N}^{\omega}$-nitro-L-arginine (L-NA) in either intact, mucosal layer removed or submucosal layer removed preparations. Small contractions were not significantly modulated by atropine or L-NA in any type of preparation. In both intact and mucosal layer removed preparations, TNS $(1 \mathrm{~Hz}$ frequency, for 2-3 min) increased the amplitude and/or frequency of large contractions, but caused no significant change in small contractions. TNS also increased the amplitude and/or frequency of large contractions in submucosal layer removed preparations, but did not significantly modulate small contractions in longitudinal muscle removed preparations. Exogenously applied acetylcholine $\left(>10^{-7} \mathrm{M}\right)$ enhanced, while sodium nitroprusside $\left(>10^{-8} \mathrm{M}\right)$ inhibited, the amplitude and frequency of both types of contraction, in a concentration-dependent manner. The results suggest that the large contractions were elicited by interstitial cells distributed in the myenteric layer (ICC-MP), while the small contractions were elicited by ICC distributed in the submuscular plexus layer (ICC-SMP). Intramural nerves mainly modulated the activity of ICC-MP, while neural regulation of ICC-SMP was weak.
\end{abstract}

Key words: spontaneous contraction, transmural nerve stimulation, ICC, pacemaker area, colon 


\section{Introduction}

Gastrointestinal smooth muscle is spontaneously active, with rhythmic generation of phasic contractions, triggered by interstitial cells of Cajal (ICC) distributed in the gut wall (Sanders, 1996; Huizinga et al., 1997). The distribution and properties of ICC are heterogeneous in the gastrointestinal tract; myenteric ICC (ICC-MP) are distributed in the myenteric layer between circular and longitudinal smooth muscle layers, intramuscular ICC (ICC-IM) are found in both the circular and longitudinal smooth muscle layers of the stomach and colon of many species, while deep muscular ICC (ICC-DMP) are distributed in the circular smooth muscle layer close to the mucosal layer of the small intestine (Komuro, 2006). In the colon, a group of ICC is distributed in the submucosal layer (submuscular ICC, ICC-SMP), in addition to both ICC-MP and ICC-IM in the guinea-pig (Kobayashi et al., 1996; Ishikawa and Komuro, 1996; Toma et al., 1999), dog (Kobayashi et al., 1995) and rat (Plujà et al., 2001). The distribution of serosal ICC (ICC-SS) is also noted in the proximal region of the guinea-pig colon (Toma et al., 1999; Aranishi et al., 2009).

The physiological roles of ICC vary between regions in the gastrointestinal tract and also between species; ICC-MP may provide the pacemaker activity for the smooth muscle of the stomach and small intestine of many species (Sanders et al., 1996). However in the colon, rhythmic activity of smooth muscle is paced by ICC-SMP in the dog (Smith et al., 1987; Kobayashi et al., 1995) and guinea-pig (Kobayashi et al., 1996; Nahar et al., 1998), while it is carried out by ICC-MP in the rat (Plujà et al., 2001; Kato et al., 2009) and mouse (Yoneda et al., 2004). In the guinea-pig, a group of ICC-SMP with a dense distribution in the flexure region at the boundary between proximal and distal divisions of colon produces regular periodic activity of 10-12 cycle per min in the circular smooth muscle (Kobayashi et al., 1995; Nahar et al., 1996). The region in which these ICC-SMP are distributed is suggested to be the pacemaker area from which the peristaltic and antiperistaltic activities of the distal and proximal regions of colon arise respectively (Hukuhara and Neya, 1968).

In circular smooth muscle preparations isolated from the proximal colon of the rat, an analysis of the mechanical responses elicited by transmural nerve stimulation (TNS) indicates that there are at least 4 different types of motor innervation, with cholinergic excitatory, peptidergic excitatory, nitrergic inhibitory and non-adrenergic non-cholinergic non-nitrergic (NANCNN) inhibitory nerves. The ICC-MP-mediated phasic contractions are strongly enhanced by cholinergic excitatory nerves and inhibited by nitrergic nerves, with only weak neural regulation of phasic contractions triggered by ICC-SMP (Kato et al., 2009). Thus, the neural regulatory mechanisms of the activity seem to differ between the different subtypes of ICC.

Attempts were made to investigate the properties of the mechanical responses produced by nerve stimulation in circular smooth muscle preparations isolated from the "pacemaker area" (Kobayashi et al., 1996) distributed in the flexure regions between proximal and distal colon of the guinea-pig. In view of the heterogeneous contribution of different types of ICC to the rhythmic activity of the colon (Kobayashi et al., 1995; Nahar et al., 1998; Plujà et al., 2001; Kato et al., 2009), it was expected that individual nerve excitation would elicit different or differential responses in both smooth muscle cells and ICC. Experiments were thus carried out to investigate the properties of the mechanical responses elicited by transmural nerve stimulation (TNS) in circular 
smooth muscle preparations isolated from the pacemaker area. The possible causal relationship between the activity of smooth muscle and the types of ICC was investigated by using several types of preparations in which either the mucosal, submucosal or longitudinal smooth muscle layers had been removed. The results indicate that the circular muscle of the pacemaker area of the colon produces two types of spontaneous contraction, one originating from ICC-MP and the other from ICC-SMP. TNS modulates the former activity alone, in both excitatory and inhibitory ways, with no significant effect on the latter.

\section{Materials and Methods}

Male guinea-pigs, weighing 200-300 g, were anesthetized with fluoromethyl 2,2,2-trifluoro-1(trifluoromethyl) ethyl ether (sevoflurane, Maruishi Pharm., Co. Ltd., Osaka, Japan), and exsanguinated by decapitation. All animals were treated ethically according to the guiding principles for the care and use of experimental animals in the field of basic sciences, as approved by The Experimental Animal Committee of the Nagoya City University Medical School. The pacemaker area distributed in the flexure region between the proximal and distal colon was excised as a 1-2 cm long segment which was opened by cutting vertically. Circular muscle strip preparations, about $1 \mathrm{~mm}$ wide and $1.5 \mathrm{~cm}$ long, were made by cutting parallel to the running direction of the circular muscle. Four different types of preparation were then made: (i) with mucosal layer removed but longitudinal layers attached (mucosal layer removed preparations), (ii) with mucosal and submucosal layer removed (submucosal layer removed preparations), (iii) with longitudinal muscle removed (longitudinal muscle removed preparations), and (iv) with all layers attached (intact preparations). In some of the longitudinal muscle removed preparations, the mucosal layer was also removed (longitudinal muscle and mucosal layer removed preparations).

Both ends of each of these preparations were tied with fine threads, and suspended vertically in a cylindrical recording chamber (diameter $10 \mathrm{~mm}, 20 \mathrm{~mm}$ deep). Preparations were superfused with oxygenated Krebs solution (warmed to $36.5^{\circ} \mathrm{C}$ ), at a constant flow rate of about $3 \mathrm{~mL} / \mathrm{min}$. One thread was anchored to the bottom of the chamber, while the other end was connected to the lever of a force-transducer (TB-612T, Nihon-Kohden, Tokyo, Japan). The isometric force changes produced by the circular muscle were recorded through a pre-amplifier (AP-621G, Nihon Kohden, Tokyo, Japan), digitized using P-clamp (Axon Instruments, Foster City, CA, USA) and stored on a personal computer for later analysis. A pair of silver plates (width, $3 \mathrm{~mm}$ ) was placed on either side of the recording chamber, along the length of the muscle segment, and brief electrical current stimuli ( $0.1 \mathrm{~ms}$ duration, $20 \mathrm{~V}$ intensity) were applied to the muscle through the plates, using an electric stimulator (SEM-3013, Nihon-Kohden, Tokyo, Japan). In most experiments, muscles were stimulated with current pulses for $2-3 \mathrm{~min}$ at $0.5-1 \mathrm{~Hz}$ frequency.

The ionic composition of the Krebs solution was as follows (mM): $\mathrm{Na}^{+} 137.4, \mathrm{~K}^{+} 5.9, \mathrm{Ca}^{2+} 2.5$, $\mathrm{Mg}^{2+} 1.2, \mathrm{HCO}_{3}^{-} 15.5, \mathrm{H}_{2} \mathrm{PO}_{4}^{-} 1.2, \mathrm{Cl}^{-} 134$ and glucose 11.5 . The solution was aerated with $\mathrm{O}_{2}$ containing $5 \% \mathrm{CO}_{2}$, and the $\mathrm{pH}$ of the solutions was maintained at between 7.2-7.3.

Chemicals used were acetylcholine chloride (ACh), apamin, atropine sulphate, capsaicin, neostigmine bromide, $\mathrm{N}^{\omega}$-nitro-L-arginine (L-NA), sodium nitroprusside (SNP) and tetrodotoxin (TTX). All chemicals were purchased from Sigma-Aldrich Chemicals (St. Louis, MI, USA). These 

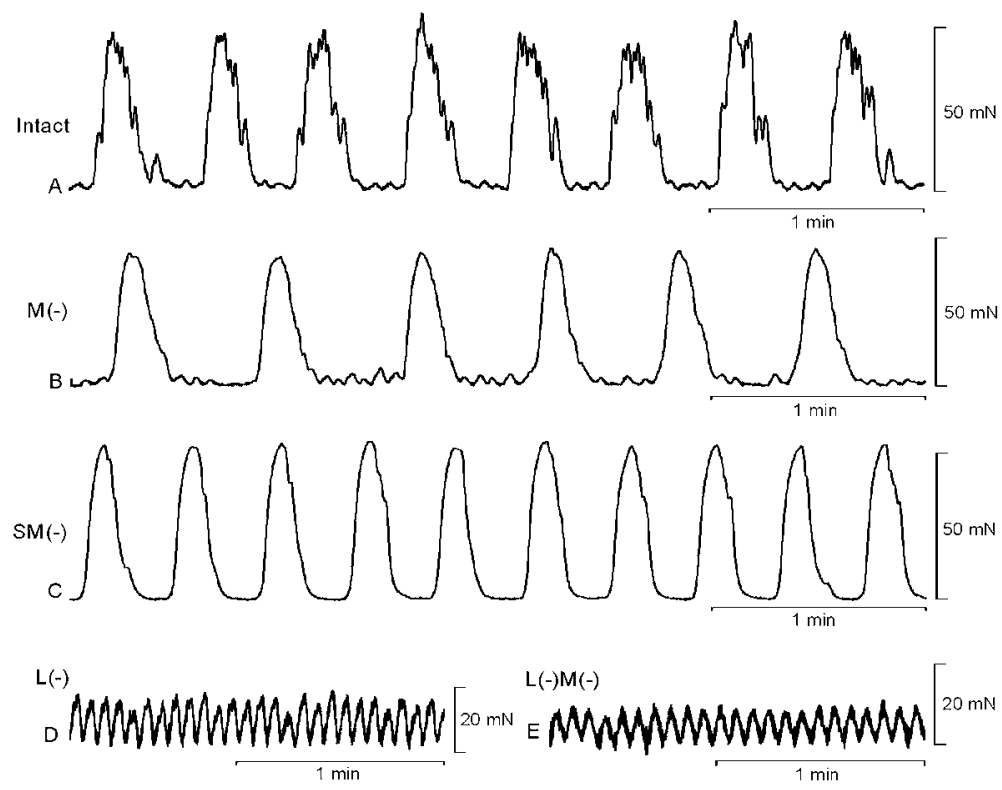

Fig. 1. Typical examples of the spontaneous mechanical activity of a circular smooth muscle preparation isolated from the P-region of the proximal colon of the guinea-pig. Responses were recorded from an intact preparation (A), a mucosal layer removed preparation (B), a submucosal layer removed preparation (C), a longitudinal muscle removed preparation (D) and a longitudinal muscle and mucosal layer removed preparation (E). All responses were recorded from different preparations.

chemicals were dissolved first in distilled water at concentrations which were more than 1,000 times higher than those used in the experiments, and then further diluted with Krebs solution to prepare the desired concentrations. The addition of these drugs did not alter the $\mathrm{pH}$ of the Krebs solution.

Experimental values were expressed as the mean value \pm standard error of the mean (SEM). Statistical significance was tested using the Student's $t$-test, and probabilities of less than $5 \%$ $(P<0.05)$ were considered to be significant.

\section{Results}

\section{Properties of spontaneous mechanical activities}

Circular smooth muscle preparations isolated from the pacemaker area of the guinea-pig colon were spontaneously active with an irregular generation of two types of phasic contractions. Large contractions were generated in intact preparations, mucosal layer removed preparations and submucosal layer removed preparations, while small contractions were observed in intact preparations, mucosal layer removed preparations and longitudinal muscle removed preparations. Typical spontaneous mechanical responses recorded from different preparations are shown in Fig. 1. In intact and mucosal layer removed preparations, both large and small contractions were generated, but the occurrence of large contractions was much lower than that of small 

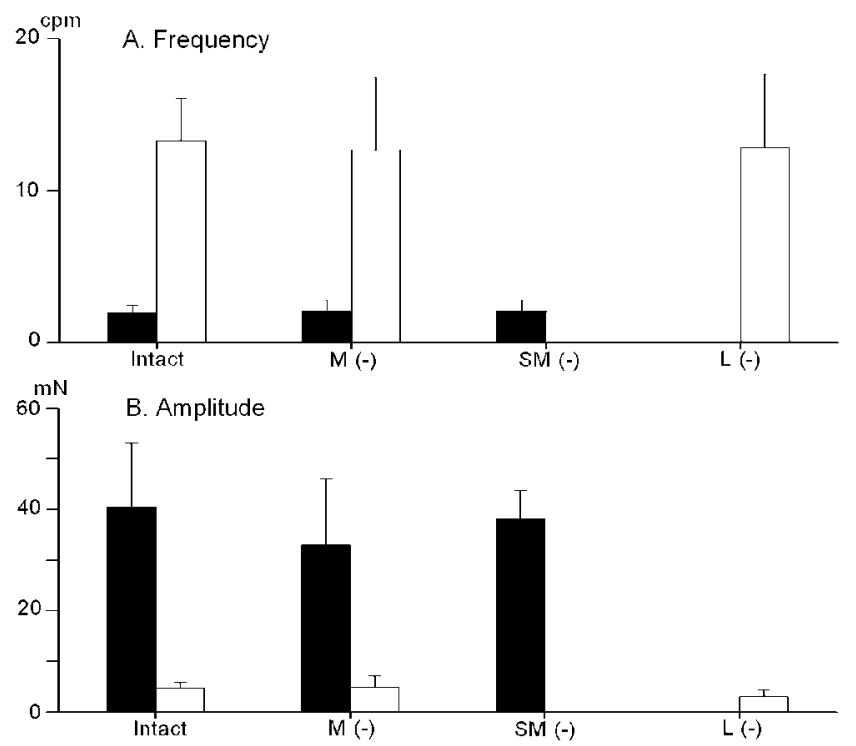

Fig. 2. Summary of the frequency (A) and amplitude (B) of phasic contractions recorded from the different types of circular smooth muscle preparations isolated from the P-region of the proximal colon of the guinea-pig. Filled column, large contractions; open column, small contractions. Intact, intact preparation; M (-), mucosal layer removed preparation; SM (-), submucosal layer removed preparation; L (-), longitudinal muscle removed preparation. Mean \pm S.D. of 5-12 preparations are shown.

contractions. The small contractions could be seen in the interval between large contractions (Fig. 1B), or they were often superimposed on the large contractions (Fig. 1A). These properties of the phasic contractions were independent of the type of preparation, and were seen in both intact and mucosal layer removed preparations. The quantified data obtained from 10-15 preparations indicated that the amplitude and frequency of the large contractions were about $40 \mathrm{mN}$ and 2 cycles per min (cpm), respectively, for both intact and mucosal layer removed preparations (Fig. 2). The amplitude (about $5 \mathrm{mN}$ ) and frequency (about $13 \mathrm{cpm}$ ) of small contractions were also similar for both intact and mucosal layer removed preparations $(P>0.05)$. No significant difference was found between intact and mucosal layer removed preparations, for both amplitude and frequency of phasic contractions, and these data suggest that the mucosal layer did not have any significant effect on spontaneous contractions. In submucosal layer removed preparations, only large contractions were generated (Fig. 1C), with an amplitude and frequency similar to those generated in intact or mucosal layer removed preparations $(P>0.05$, Fig. 2$)$.

The longitudinal muscle removed preparations only generated small contractions (Fig. 1D), with an amplitude and frequency not significantly different from those generated in both intact and mucosal layer removed preparations $(P>0.05$, Fig. 2$)$. Attempts were made to observe the effects of removal of the mucosal layer on the small contractions, in 3 longitudinal muscle layer removed preparations. Preparations with both longitudinal muscle and mucosal layers removed again generated small contractions with similar amplitude and frequency (Fig. 1E; longitudinal muscle removed preparations: amplitude $3.1 \pm 1.4 \mathrm{mN}$, frequency $12.8 \pm 4.8 \mathrm{cpm}, \mathrm{n}=3$; longitudinal 

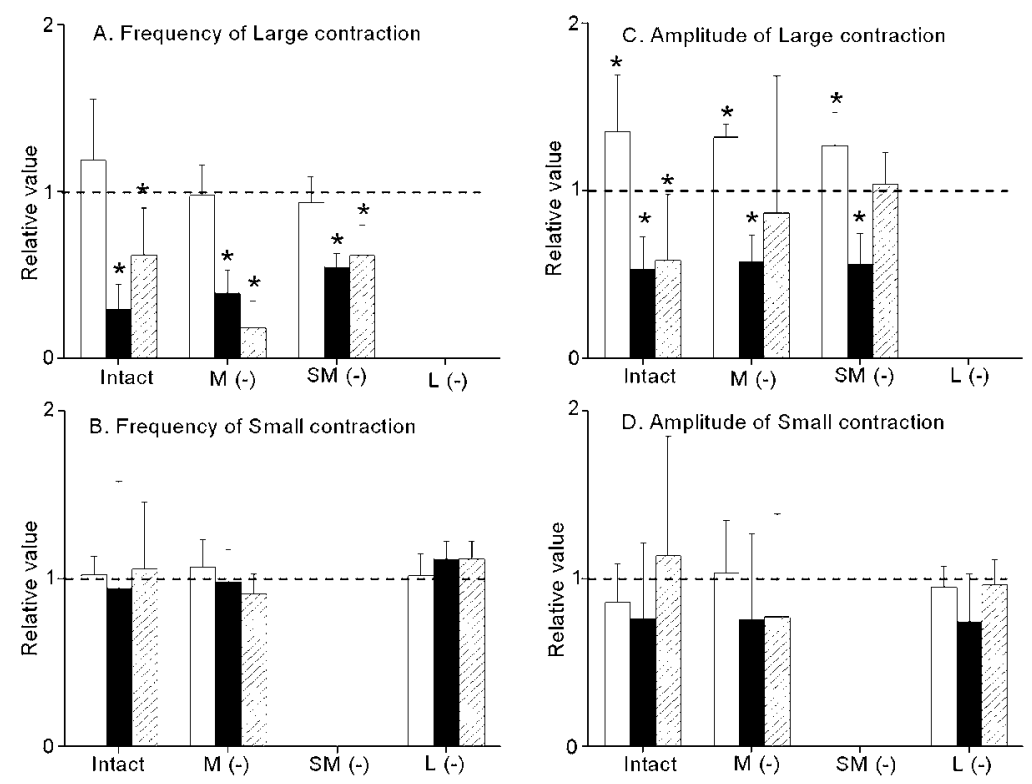

Fig. 3. Effects of atropine and L-NA on the spontaneous contractions recorded from circular muscle preparations isolated from the P-region of the proximal colon of the guinea-pig. Amplitude and frequency of phasic contractions generated in the presence of $3 \times 10^{-5} \mathrm{M}$ L-NA (open column), $10^{-6} \mathrm{M}$ atropine (filled column) and $10^{-6} \mathrm{M}$ atropine plus $3 \times 10^{-5} \mathrm{M}$ L-NA (oblique line column) are show as relative to those generated before application of these inhibitors. A, frequency of large contractions; B, frequency of small contractions; $\mathrm{C}$, amplitude of large contractions; $\mathrm{D}$, amplitude of small contractions. Intact, intact preparation; M (-), mucosal layer removed preparation; SM (-), submucosal layer removed preparation; L (-), longitudinal muscle removed preparation. Mean \pm SD $(\mathrm{n}=5-12)$ are shown. *, significantly different from control $(P<0.05)$.

muscle and mucosal layer removed preparations: amplitude $3.3 \pm 1.6 \mathrm{mN}$, frequency $13.1 \pm 3.5$ cpm, $\mathrm{n}=3 ; P>0.05$ for both). These results again suggest that the mucosal layer made no significant contribution to the generation of the small contractions.

\section{Effects of $N^{\omega}$-nitro-L-arginine and atropine on spontaneous phasic contractions}

Experiments were carried out to observe the effects of $\mathrm{N}^{\omega}$-nitro-L-arginine (L-NA), an inhibitor of the production of nitric oxide (NO), and atropine, an inhibitor of muscarinic receptors, on spontaneous phasic contractions in circular muscle preparations isolated from the pacemaker area. The amplitude and frequency of phasic contractions varied between preparations, for both large and small contractions. Therefore, a comparison was made of the amplitude and frequency of the phasic contractions generated in the presence of L-NA $\left(3 \times 10^{-5} \mathrm{M}\right)$, atropine $\left(10^{-6} \mathrm{M}\right)$ or both, as relative to those generated in the absence of these chemicals. Measurement of phasic contraction activity was made after exposure of the preparations to these chemicals for more than 15 min. The results (Fig. 3) indicate that L-NA increased the amplitude but not the frequency of large contractions generated in intact, mucosal layer removed and submucosal layer removed preparations, while atropine decreased both the amplitude and frequency of large contractions. 


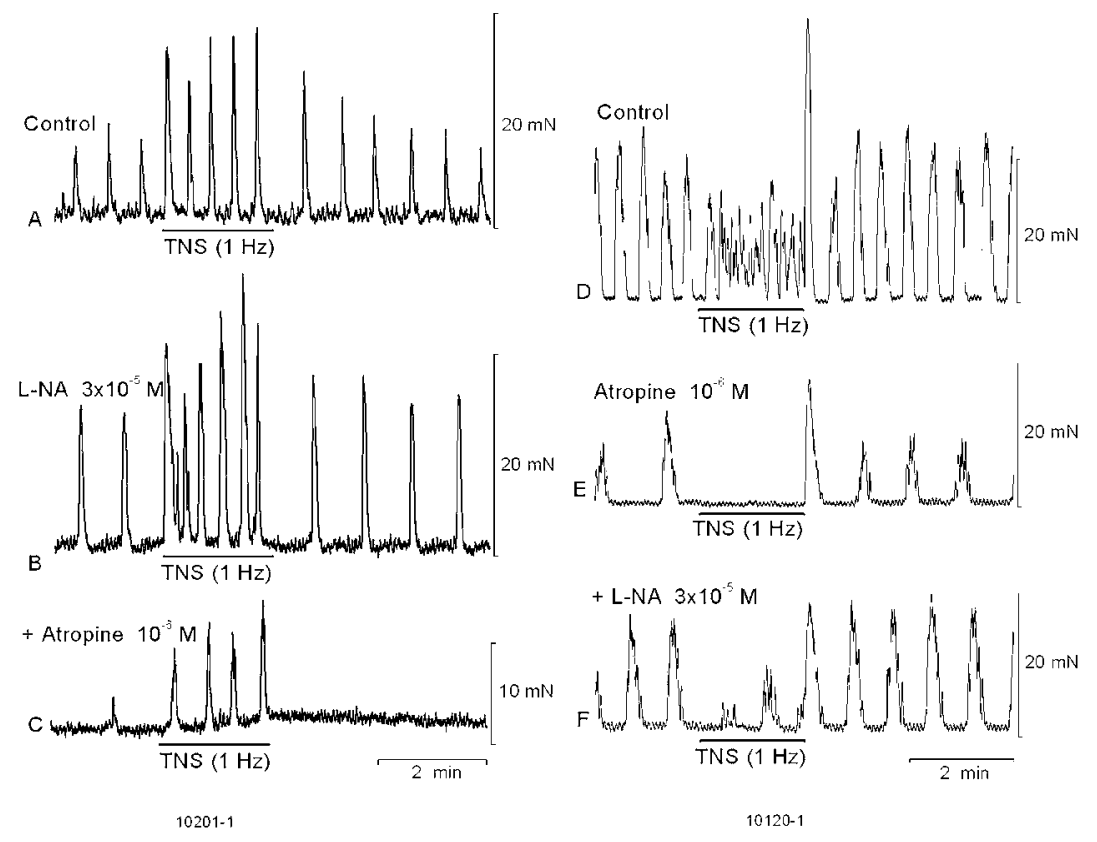

Fig. 4. Effects of transmural nerve stimulation (TNS) on phasic contractions of circular smooth muscle preparations isolated from the P-region of the proximal colon of the guinea-pig. Intact preparations. TNS ( $1 \mathrm{~Hz}$ frequency, for $2 \mathrm{~min}$ ) was applied at the bar under each trace, in the absence (Control, A and D) or presence of $3 \times 10^{-5} \mathrm{M} \mathrm{L-NA}(\mathrm{B}), 10^{-6} \mathrm{M}$ atropine (E) and both atropine and L-NA (C and F). A-C and D-F were recorded from different single preparations.

Application of both atropine and L-NA significantly inhibited the frequency and decreased the amplitude of the large contractions only in intact preparations. Both the amplitude and frequency of small contractions were not significantly modulated by atropine or L-NA, in any types of preparation (Fig. 3, B and D). The effects of atropine or L-NA on spontaneous contractions generated in preparations which had both longitudinal muscle and mucosal layers removed were not examined. These results indicated that atropine and L-NA modulated the amplitude or frequency only of the large contractions, but not of small contractions.

\section{Modulation of large contractions by transmural nerve stimulation}

Experiments were carried out to investigate the properties of the large contractions produced by direct excitation of intramural nerves using transmural application of brief electric pulses (i.e., transmural nerve stimulation, TNS) in intact, mucosal layer removed and submucosal layer removed preparations. Selective excitation by TNS of intramural nerves was confirmed by a reversible inhibition of the evoked responses with $3 \times 10^{-7} \mathrm{M}$ TTX (data not shown). In intact preparations, TNS applied at $1 \mathrm{~Hz}$ frequency for 2 min elicited an excitatory response with an increase in either amplitude or frequency, or both, of the large contractions. In 20 preparations examined, TNS increased both the amplitude and frequency of large contractions in 15 preparations (Fig. 4A), while in 5 preparations TNS increased the frequency and decreased the 

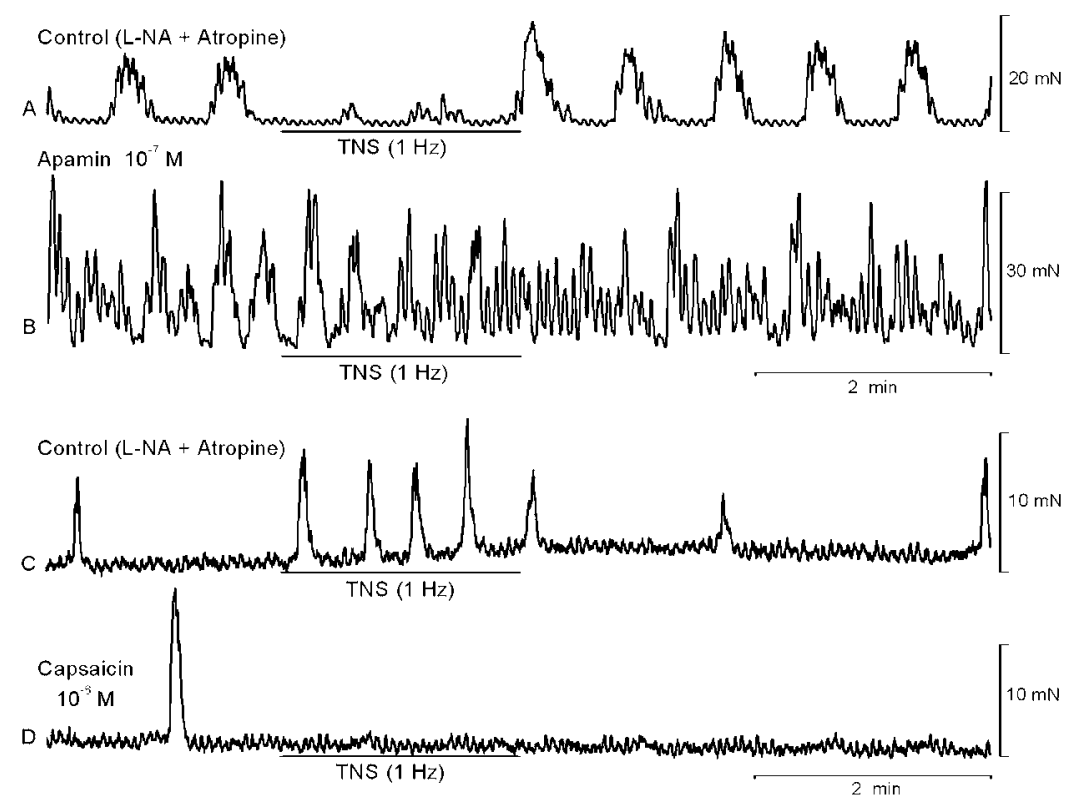

Fig. 5. Effects of apamin and capsaicin on phasic contractions modulated by TNS in intact circular muscle preparations isolated from the P-region of the proximal colon of the guinea-pig. TNS ( $1 \mathrm{~Hz}$ frequency, for $2 \mathrm{~min}$ ) was applied at the bar under each trace. TNS-induced responses were recorded in the presence of $10^{-6} \mathrm{M}$ atropine and $3 \times 10^{-5}$ M L-NA (Control, A and C) and additional application of $10^{-7} \mathrm{M}$ apamin (B) or $10^{-6} \mathrm{M}$ capsaicin (D). A-B and C-D were recorded from different single preparations.

amplitude of large contractions (Fig. 4D). Application of L-NA $\left(3 \times 10^{-5} \mathrm{M}\right)$ enhanced the TNSinduced excitatory responses (Fig. 4B), while the responses were inhibited by $10^{-6} \mathrm{M}$ atropine (Fig. 4C). In preparations in which TNS elicited an increase in frequency and a decrease in amplitude of large contractions, application of L-NA produced an increase in both amplitude and frequency of the large contractions ( $n=2$, data not shown). Attempts were made to investigate the effects of first an application of atropine, followed by an application of L-NA, on the change in properties of TNS-induced responses. In preparations which showed excitatory responses to TNS, application of atropine resulted in an inhibition of the generation of large contractions by TNS (Fig. $4 \mathrm{E})$. The TNS-induced inhibition of the generation of large contractions was attenuated by additional application of L-NA (Fig. 4F).

In intact preparations which had been exposed to L-NA and atropine, TNS elicited either excitatory responses (12 preparations) or inhibitory responses (6 preparations), with the remainder (2 preparations) producing no response. Experiments were carried out to investigate further the effects of apamin $\left(10^{-7} \mathrm{M}\right)$ or capsaicin $\left(10^{-6} \mathrm{M}\right)$ on the inhibitory or excitatory responses elicited by TNS in the presence of either L-NA or atropine. Application of apamin increased the amplitude of the small contractions, and often elicited the generation of contractions which were difficult to identify as either large or small contractions. In the presence of apamin, TNS elicited no clear response (Fig. 5B). Exposure of preparations to capsaicin reduced spontaneous activity, after a transient increase in the generation of large contractions (data not 


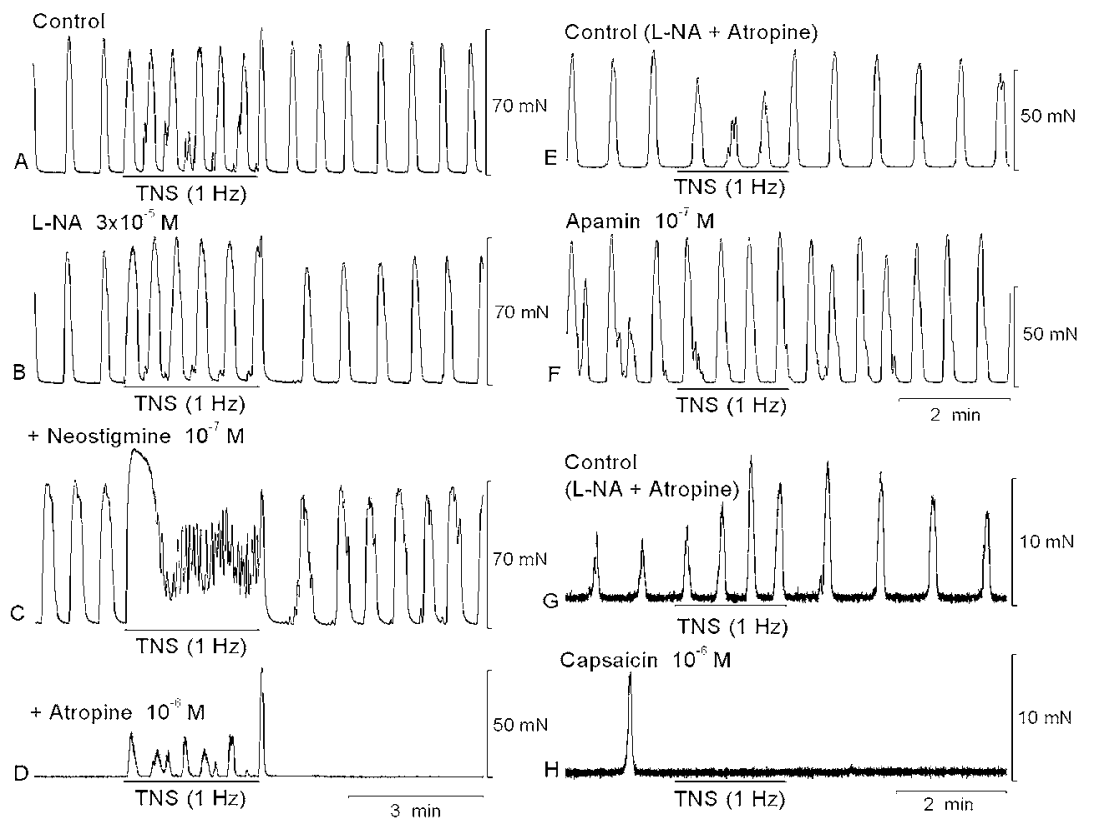

Fig. 6. Mechanical responses produced by TNS in submucosal layer removed preparations. TNS ( $1 \mathrm{~Hz}$ frequency, for $2 \mathrm{~min}$ ) was applied at the bar under each trace. The responses were recorded before (A, Control) and after cumulative application of $3 \times 10^{-5} \mathrm{M} \mathrm{L-NA}$ (B), $10^{-7} \mathrm{M}$ neostigmine (C) and $10^{-6} \mathrm{M}$ atropine (D). TNS-induced responses were recorded in the presence of atropine and L-NA (E, G) and additional application of $10^{-7}$ $\mathrm{M}$ apamin $(\mathrm{F})$ or $10^{-6} \mathrm{M}$ capsaicin $(\mathrm{H})$. A-D, E-F, and G-H were recorded from different single preparations.

shown), while TNS applied in the presence of capsaicin produced no clear mechanical response (Fig. 5D). Thus, the results showed that the TNS-induced inhibitory and excitatory responses that were sensitive to apamin and capsaicin, respectively. It is also worthwhile to note that TNS applied in the presence of apamin or capsaicin again did not modulate the small contractions, although their amplitude was increased by apamin (see Fig. 5, A and B).

The effects of TNS on large contractions, and their modulation by L-NA and atropine, were examined in submucosal layer removed preparations. In the absence of L-NA and atropine, TNS elicited an excitatory response with an increase in either the amplitude or frequency, or both, of the large contractions (Fig. 6A). The TNS-induced excitatory responses were enhanced by L-NA (Fig. $6 \mathrm{~B}$ ), and were further enhanced by neostigmine ( $10^{-7} \mathrm{M}$, Fig. $\left.6 \mathrm{C}\right)$. Atropine inhibited the frequency and amplitude of the large contractions (Fig. 3), with TNS producing either excitatory (Fig. 6, D and G) or inhibitory responses (Fig. 6E). The TNS-induced inhibitory responses were blocked by apamin (Fig. 6F), while the TNS-induced excitatory responses were blocked by capsaicin (Fig. 6H).

These results suggest that the TNS-induced excitatory responses are mediated by an atropine-sensitive muscarinic receptor, suggesting that they are produced by acetylcholine (ACh) released from cholinergic nerves. The TNS-induced inhibitory responses may be produced by an L-NA-sensitive mechanism, possibly due to an elevated production of neurogenic NO. If this is the 

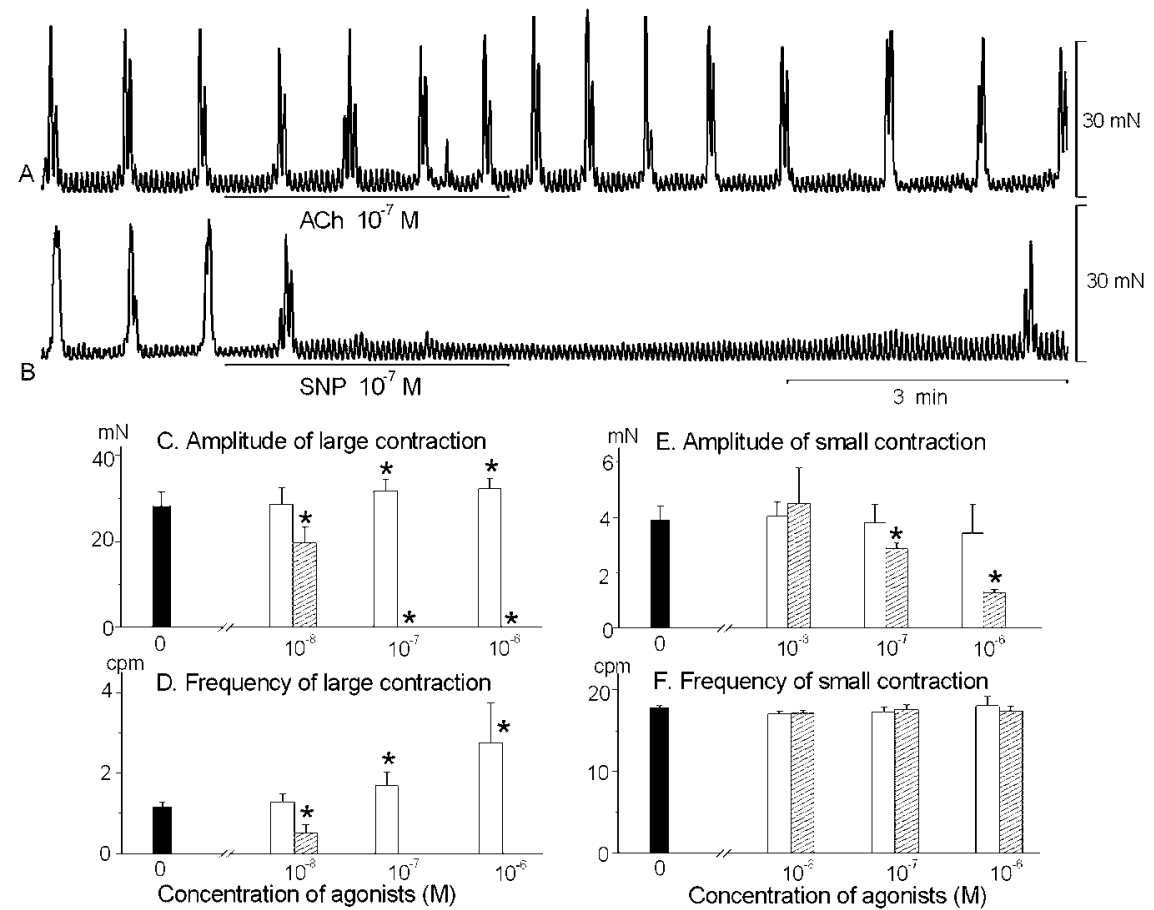

Fig. 7. Mechanical responses elicited by $\mathrm{ACh}$ or sodium nitroprusside (SNP) in intact preparations isolated from the pacemaker area of the guinea-pig proximal colon. ACh $\left(\mathrm{A}, 10^{-7} \mathrm{M}\right)$ or SNP $\left(\mathrm{B}, 10^{-7} \mathrm{M}\right)$ was applied for $3 \mathrm{~min}$, at the bar under each trace. A and $\mathrm{B}$ were recorded from the same preparation. Amplitude $(\mathrm{C}, \mathrm{E})$ and frequency $(\mathrm{D}$, $\mathrm{F})$ of large $(\mathrm{C}, \mathrm{D})$ and small phasic contractions $(\mathrm{E}, \mathrm{F})$ obtained in the absence (filled column) and the presence of different concentrations $\left(10^{-8}-10^{-6} \mathrm{M}\right)$ of agonist (ACh, open column; SNP, oblique line column) are shown by the mean values + SEM $(n=5-20)$. *, significantly different from values obtained in the absence of agonists $(P<0.05)$.

case, it would be expected that exogenously applied ACh would elicit excitatory effects while NO would elicit inhibitory effects on the large contractions. Figure $7 \mathrm{~A}$ shows that $\mathrm{ACh}\left(10^{-7} \mathrm{M}\right)$ increased the amplitude and frequency of large contractions, while exogenously applied sodium nitroprusside (SNP, $10^{-7} \mathrm{M}$ ), an NO donor, produced an inhibition of the generation of large contractions in intact preparations (Fig. 7B), with no clear change in the activity of the small contractions. The pooled data obtained from different preparations indicates that $\mathrm{ACh}\left(>10^{-7} \mathrm{M}\right)$ increased both the amplitude (Fig. 7C) and the frequency (Fig. 7D) of the large contractions, in a concentration-dependent manner, with no significant change in either the amplitude (Fig. 7E) or frequency (Fig. $7 \mathrm{~F})$ of the small contractions. SNP $\left(10^{-8} \mathrm{M}\right)$ reduced the amplitude and frequency of the large contractions, while higher concentrations of SNP $\left(>10^{-7} \mathrm{M}\right)$ abolished large contractions (Fig. 7, C and D). The amplitude of small contractions was reduced by SNP $\left(>10^{-7} \mathrm{M}\right)$ in a concentration-dependent manner (Fig. 7E), however the frequency of small contractions was not significant modulated by $10^{-8}-10^{-6} \mathrm{M}$ SNP (Fig. $7 \mathrm{~F}$ ). 


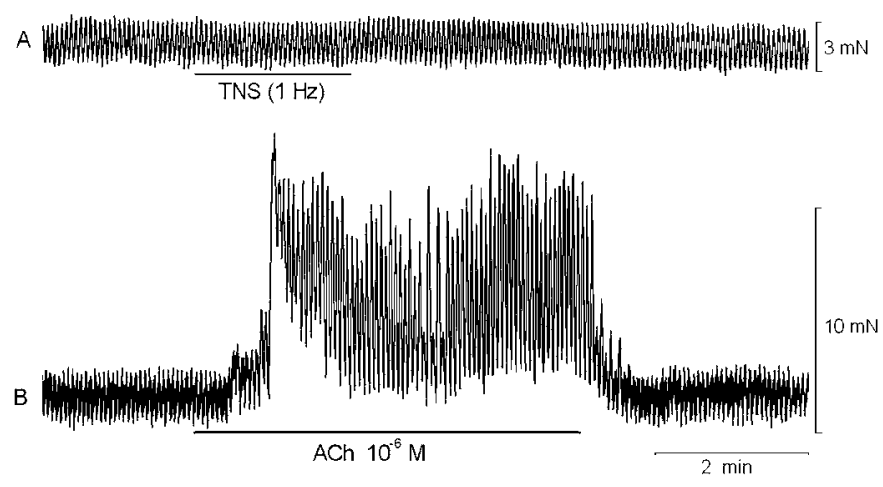

Fig. 8. Mechanical responses produced by TNS and exogenously applied ACh in longitudinal muscle removed preparations. Responses were elicited by TNS ( $1 \mathrm{~Hz}$, for $2 \mathrm{~min}$ ) (A) or $10^{-6} \mathrm{M} \mathrm{ACh}(\mathrm{B})$. A and B were recorded from same preparation. TNS or ACh was applied at the bar under each trace.

\section{Modulation of small contractions by TNS}

The effects of TNS on small contractions were examined in intact and longitudinal muscle removed preparations. In both preparations, both the frequency and amplitude of the small contractions were not modulated by TNS. A typical example of the effects of TNS on the small contractions generated in the longitudinal muscle removed preparations indicates that no detectable change is elicited by TNS (Fig. 8A). Attempts were made to stimulate longitudinal muscle preparations with exogenously applied ACh. As shown in Figure 8B, exogenously applied ACh $\left(10^{-6} \mathrm{M}\right)$ could increased the amplitude of small contractions (control, $2.39 \pm 0.17 \mathrm{mN}$; in ACh $6.68 \pm 1.17 \mathrm{mN} ; \mathrm{n}=4, P<0.05$ ), but again with no significant change in the frequency (control, $15.35 \pm 0.44 \mathrm{cpm}$; in $\mathrm{ACh}, 15.79 \pm 0.63 \mathrm{cpm} ; \mathrm{n}=4, P>0.05$; Fig. $8 \mathrm{~B})$. The results suggest that in circular smooth muscle with no attached longitudinal muscle, excitatory muscarinic receptors were functional.

\section{Discussion}

The present experiments revealed that there were two types of periodic phasic contraction in circular smooth muscle preparations isolated from the pacemaker area of the flexure region of the guinea-pig colon. There were large contractions with a frequency of about $2 \mathrm{cpm}$ and small contractions with a frequency of about $13 \mathrm{cpm}$. The amplitude of the large contractions was several times larger than that of the small contractions. The properties of the contractions generated in preparations which had either the submucosal layer or longitudinal muscle removed revealed that the generation of large contractions required an attached longitudinal muscle layer but not an attached submucosal layer, while the generation of small contractions required an attached submucosal layer but not an attached longitudinal muscle layer. The pacemaker area has both ICC-MP distributed in the myenteric layer and ICC-SMP distributed in the submucosal layer (Kobayashi et al., 1996; Nahar et al., 1998), in addition to ICC-IM in both the circular and longitudinal smooth muscle layers (Toma et al., 1999). The removal of the longitudinal smooth 
muscle layer is associated with the loss of the myenteric layer from the circular smooth muscle layer (Kodama et al., 2010). Thus, the preparations which had the submucosal layer removed contained only ICC-MP, which produced the large contractions. The results also suggested that the small contractions were produced by activity generated by ICC-SMP distributed in the submucosal layer. These differential contributions by ICC-MY and ICC-SMP on the activity of circular smooth muscle were similar to those found in the proximal colon of both the rat (Plujà $e t$ al., 1999; Kato et al., 2009) and the mouse (Yoneda et al., 2004). Our results also confirmed the contribution of ICC-SMP on the generation of the high-frequency contraction of circular muscle in the pacemaker area of the guinea-pig colon (Kobayashi et al., 1996; Nahar et al., 1998).

Both ICC-MP and ICC-SMP have gap junctional connections to smooth muscle cells (Daniel and Wang, 1999), and functional connections between these cells has been suggested. Both the electrical (Plujà et al., 1995) and mechanical responses (Plujà et al., 1995; Kato et al., 2009) of circular smooth muscle cells have been shown to originate from both the ICC-MP and the ICCSMP. The present experiments have indicated that in intact or mucosal layer removed preparations, both large and small contractions appeared together in the circular smooth muscle preparations, indicating that both ICC-MP and ICC-SMP may be electrically connected indirectly through circular smooth muscle cells. However, the results have also indicated that the frequency of the large contractions was much lower than that of the small contractions, and it did not differ significantly between intact and submucosal layer removed preparations. These results suggest that the activity of the ICC-MP is independent of that of the ICC-SMP. In the mouse, ICC-SMP produce plateau-type action potentials which are conducted to circular smooth muscle cells, possibly in an electrotonic manner, to elicit action potentials (Yoneda et al., 2002; Yoneda et al., 2003; Hotta et al., 2007). It was therefore considered that the activity propagated from one type of ICC could not be conducted to other types of ICC, or alternatively the activity propagated from one type of ICC was insufficient to modulate the activity of other types of ICC.

The present results also indicate that the activities of both ICC-MP and ICC-SMP were independent of the mucosal layer, as the removal of this layer did not significantly result in alteration of the frequency of the spontaneous contractions. Some myenteric nerves extend their axons into the mucosal layers, such as the Dogiel type II fibers (Kunze and Furness 1999; Furness, 2000; Lomax and Furness, 2000), and some of these could terminate on ICC-SMP distributed in the submucosal layer. There is a rich distribution of nerve terminals around ICC-SMP in the guinea-pig colon (Ishikawa and Komuro, 1996; Toma et al., 1999). However, experiments using transmural nerve stimulation (TMS) indicated that there was no distribution of functional nerves to the ICC-SMP. These results suggest that the mucosal layer is not important for the activity of ICC in the proximal colon of the guinea-pig.

The properties of the neural regulation of rhythmic contraction were examined using TNS, with the results indicating that large contractions are regulated by excitatory nerves which increased the frequency or amplitude, or both, of these phasic contractions. The responses were augmented by neostigmine and antagonized by atropine, indicating that the main transmitter substance was acetylcholine (ACh), further supported by the simulation of TNS-induced responses by exogenously applied ACh. In the presence of atropine, TNS-induced inhibitory responses were attenuated by L-NA, suggesting that the inhibition was mediated by NO. In the presence of both 
atropine and L-NA, there were some preparations which produced either excitatory or inhibitory responses during TNS, with the former being antagonized by capsaicin or the latter by apamin. Gastrointestinal smooth muscle has a rich distribution of excitatory peptidergic nerves which release substance P in response to TNS (Hoyle and Burnstock, 1989). It was therefore suggested that the capsaicin-sensitive responses were produced by substance $\mathrm{P}$ released from peptidergic nerves (or substance P-ergic nerves), while an involvement of unidentified substances released from non-adrenergic non-cholinergic non-nitrergic (NANCNN) inhibitory nerves was suggested in the apamin-sensitive responses. From these results, it was deduced that pacemaker cells and possibly the related smooth muscle cells contributing to the large contractions were innervated by cholinergic excitatory nerves, nitrergic inhibitory nerves, substance P-ergic excitatory nerves and NANCNN inhibitory nerves. These results agree with the type of neurons involved in gastrointestinal smooth muscle tissue (Hoyle and Burnstock, 1989; Furness et al., 1994; Kuriyama et al., 1998; Kunze and Furness 1999; Furness, 2000; Lomax and Furness, 2000), and were also in agreement with the results examined in the proximal region but not the pacemaker area of the guinea-pig colon (Kodama et al., 2010). The density of the nerves distributed in individual preparations seemed to be non-homogenous, and in general the former two were high, compared to the latter two. Such heterogeneity in the density of effective nerve distribution between preparations would be causally related to the appearance of two types of excitatory responses, an increase in frequency of large contractions which was associated with an increase in amplitude of contractions (Fig. 4A)or a decrease in amplitude of large contractions with associated increase in the frequency (Fig. 4D). In the former, the TNS-induced increase in amplitude and frequency of large contractions was further enhanced by L-NA, while in the latter the TNS-induced decrease in amplitude of large contractions were changed to an increased amplitude by application of L-NA, with an associated increase in the frequency of contractions in both cases. These results could be interpreted by assuming that the density (or amount) of nitrergic inhibitory nerves varied between preparations, and the former had little content and the latter had large amount of the content of nitrergic nerves.

Small contractions, possibly generated in response to the activity of the ICC-SMP, were not modulated by TNS, in any type of preparation. TNS did not modulate the small contractions, either in the absence or presence of L-NA, suggesting that the absence of response to TNS was not the result of a balance between excitatory cholinergic and inhibitory nitrergic inputs. Furthermore, exogenously applied ACh increased and SNP decreased the amplitude of the small contractions, with no marked change in the frequency, in both intact and longitudinal muscle removed preparations. It is considered that the changes in amplitude of the phasic contractions may reflect responses of smooth muscle cells, while the changes in frequency of contractions may reflect the responses of ICC. In this case, exogenously applied agonists will modulate only the amplitude of contractions with no significant change in the frequency, if they stimulate only smooth muscle cells. This was indeed the case observed in the present experiments (Fig. 7). Similar differential responses of smooth muscle cells and ICC have also been observed when gastric smooth muscle preparations are exposed to hypoxic conditions (Nakamura et al., 2009). These results suggest that the activity of ICC-SMP are not regulated by cholinergic and nitrergic nerves. These observations sound peculiar from a histological point of view, as a rich innervation of ICC-SMP 
exists, but not of ICC-MP (Ishikawa and Komuro, 1996; Toma et al., 1999). In the stomach and intestine, either ICC-IM or ICC-DMP have a role in the mediation of the neural signals to smooth muscle cells (Ward and Sanders, 2001). This hypothesis is supported by the dense distribution of nerve terminals around these ICC (Zhou and Komuro, 1992; Furness et al., 1994; Toma et al., 1999).

Spontaneous contractions were modulated by atropine and L-NA, in the absence of TNS application. This suggests that there is a continuous stimulation of smooth muscle cells and ICC by both $\mathrm{ACh}$ and $\mathrm{NO}$, possibly released from intramuscular nerves. The release of $\mathrm{ACh}$ in the absence of TNS was also suggested from the enhanced amplitude of contractions as a result of the inhibition of the activity of acetylcholine esterase with neostigmine. Atropine decreased and L-NA increased the amplitude and frequency of the large contractions, with no significant change in the small contractions. These results again suggest that ICC-MP are neurally regulated, while ICCSMP are not. It is proposed that in the intestine, functional nerves terminate on ICC-IM or ICCDMP, and responses (junction potentials) evoked in these ICC are conducted to smooth muscle cells in an electrotonic manner through gap junctions (Ward and Sanders, 2001). It is therefore considered that there is an absence of functional nerves to the ICC-SMP distributed in the pacemaker area of the guinea-pig colon.

It is summarized that in the pacemaker area of the flexure region of the guinea-pig colon, phasic contractions of circular muscle are produced by different types of ICC. The large contractions are triggered by ICC-MP distributed in the myenteric layer and the small contractions are triggered by ICC-SMP distributed in the submucosal layer. TNS experiments indicated that the activity of the ICC-MP is modulated by cholinergic and peptidergic excitatory nerves and nitrergic and NANCNN inhibitory nerves. The density of these nerves is not homogenous, and cholinergic excitatory and nitrergic inhibitory nerves have the dominant function. Neural regulation of the activity of ICC-SMP which produce small phasic contractions seems to be very weak.

\section{Acknowledgements}

This research was supported by a Grant-in-Aid for Scientific Research from the Ministry of Education, Science, Technology and Sports of Japan to H.S. (21590237). The authors thank Prof. Terumasa Komuro, Waseda University, for the suggestions on the technical terminology of ICC in gastrointestinal tissues.

\section{References}

Aranishi, H., Kunisawa, Y. and Komuro, T. (2009). Characterization of interstitial cells of Cajal in the subserosal layer of the guinea-pig colon. Cell Tissue Res. 335: 323-329.

Daniel, E.E. and Wang, Y.E. (1999). Gap junctions in intestinal smooth muscle and interstitial cells of Cajal. Microsc. Res. Tech. 47: 309-320.

Furness, J.B., Bornstein, J.C., Pompolo, S., Young, H.M., Kunze, W.A.A. and Kelly, H. (1994). The circuitry of the enteric nervous system. Neurogastroenterol. Mot. 6: 241-253.

Hotta, A., Okada, N. and Suzuki, H. (2007). Mibefradil-sensitive component involved in the plateau 
potential in submucosal interstitial cells of the murine proximal colon. Biochem. Biophys. Res. Comm. 353: 170-176.

Hoyle, C.H.V. and Burnstock, G. (1989). Neuromuscular transmission in the gastrointestinal tract. Handbook of Physiology 6, The Gastrointestinal System, Vol. 1, Part 1, Am. Physiol. Soc. Bethesda, pp. 435-464.

Huizinga, J.D., Thuneberg, L., Vanderwinden, J.M. and Rumessen, J. (1997). Interstitial cells of Cajal as targets for pharmacological intervention in gastrointestinal motor disorders. Trends Pharmacol. Sci. 18: 393-403.

Hukuhara, T. and Neya, T. (1968). The movements of the colon of rats and guinea pigs. Jpn. J. Physiol. 18: 551-562.

Ishikawa, K. and Komuro, T. (1996). Characterization of the interstitial cells associated with the submuscular plexus of the guinea-pig colon. Anat. Embriol. 194: 49-55.

Kato, T., Nakamura, E., Imaeda, K. and Suzuki, H. (2009). Modulation of the activity of two pacemakers by transmural nerve stimulation in circular smooth muscle preparations isolated from the rat proximal colon. J. Smooth Muscle Res. 45: 249-268.

Kobayashi, S., Chowdhury, J.U., Tokuno, H., Nahar, S. and Iino, S. (1996). A smooth muscle nodule producing 10-12 cycles/min regular contractions at the mesenteric border of the pacemaker area in the guinea-pig colon. Arch. Histol. Cytol. 59: 159-168.

Kobayashi, S., Torihashi, S., Iino, S., Pang, Y.W., Chowdhury, J.U. and Tomita, T. (1995). The inner sublayer of the circular muscle coat in the canine proximal colon: origins of spontaneous electrical and mechanical activity. Arch. Histol. Cytol. 58: 45-63.

Kodama, Y., Iino, S. and Suzuki, H. (2010). Properties of acetylcholine-induced relaxation of smooth muscle isolated from the proximal colon of the guinea-pig. J. Smooth Muscle Res. 46: 185-200.

Komuro, T. (2006). Structure and organization of interstitial cells of Cajal in the gastrointestinal tract. J. Physiol. (Lond.) 576: 653-658.

Kunze, W.A.A. and Furness, J.B. (1999). The enteric nervous system and regulation of intestinal motility. Annu. Rev. Physiol. 61: 117-142.

Kuriyama, H., Kitamura, K., Itoh, T. and Inoue. R. (1998). Physiological features of visceral smooth muscle cells, with special reference to receptors and ion channels. Physiol. Rev. 78: 811-920.

Lomax, A.E. and Furness, J.B. (2000). Neurochemical classification of enteric neurons in the guinea-pig distal colon. Cell Tissue Res. 302: 59-72.

Nahar, N.S., Torihashi, S., Iino, S., Senda, T., Chowdhury, J.U. and Kobayashi, S. (1998). Special smooth muscle cells along the submucosal surface of the guinea pig colon with reference to its spontaneous contractions. Cell Tissue Res. 293: 143-154.

Nakamura, E., Yokoi, T., Fukuta, H., Iida, T., Tanaka, Y., Yamamoto, Y. and Suzuki, H. (2009). Hypoxia differentially modulates the activity of pacemaker and smooth muscle cells in the guinea pig stomach antrum. J. Smooth Muscle Res. 45: 149-166.

Neunlist, M. and Schemann, M. (1997). Projections and neurochemical coding of myenteric neurons innervating the mucosa of the guinea pig proximal colon. Cell Tiss. Res. 287: 119-125.

Plujà, L., Albertí, E., Fernández, E., Mikkelsen, H.B., Thuneberg, L. and Jiménez, M. (2001). Evidence supporting presence of two pacemakers in rat colon. Am. J. Physiol. 281: G255-G266.

Sanders, K.M. (1996). A case for interstitial cells of Cajal as pacemakers and mediators of neurotransmission in the gastrointestinal tract. Gastroenterol. 111: 492-515.

Smith, T.K., Reed, J.B. and Sanders, K.M. (1987). Origin and propagation of electrical slow waves in circular smooth muscle of the canine proximal colon. Am. J. Physiol. 252: C212-C224.

Toma, H., Nakamura, K., Kuraoka, A., Tanaka, M. and Kawabuchi, M. (1999). Three-dimensional structures of c-kit-positive cellular networks in the guinea pig small intestine and colon. Cell Tissue Res. 295: 425-436.

Ward, S.M. and Sanders, K.M. (2001). Interstitial cells of Cajal: primary targets of enteric motor 
innervation. Anat. Rec. 262: 125-135.

Yoneda, S., Fukui, H. and Takaki, M. (2004). Pacemaker activity from submucosal interstitial cells of Cajal drives high-frequency and low-amplitude circular muscle contractions in the mouse proximal colon. Neurogastroenterol. Motil. 16: 621-627.

Yoneda, S., Takano, H., Takaki, M. and Suzuki, H. (2002). Properties of spontaneously active cells distributed in the submucosal layer of mouse proximal colon. J. Physiol. (Lond.) 542: 887-897.

Yoneda, S., Takano, H., Takaki, M. and Suzuki, H. (2003). Effects of nifedipine and nickel on plateau potentials generated in submucosal interstitial cells distributed in the mouse proximal colon. $J$. Smooth Muscle Res. 39: 55-65.

Zhou, D.S. and Komuro, T. (1992). Interstitial cells associated with the deep muscular plexus of the guineapig small intestine, with special reference to the interstitial cells of Cajal. Cell Tissue Res. 268: 205-216. 